Since its first demonstration in the late nineties [1], the V shape electrothermal actuator is increasingly implemented in a wide spectrum of applications [2]. This actuator is among the most common actuators in micro- and nano-electro-mechanical systems (MEMS and NEMS) due to its simplicity, monolithic and compliant structure, compatibility with common fabrication processes, large force and displacement relative to other actuators at the same scale, linear motion, parallel stacking to increase forces, scalability, low driving voltage, small size, flexibility of design, easy integration with mechanical and electronic on-chip components, reliability, and long lifetime.

The $\mathrm{V}$ shape actuator consists of several thin inclined beams that are designed as expansion elements and/are connected symmetrically to a middle shaft (shuttle). This creates a localized output lateral force and/or linear deflection at the shuttle when heating the beams. The output force and deflection are generally controlled electro-thermally using Joule's heating. Other heating modes are also possible, such as convection with a proximity parallel layer [3], using light or directive laser [4,5], or with the environment temperature $[6,7]$ (for temperature sensing).

The design of actuators in general is more robust and accurate when it relies on accurate models. Several models were proposed for the $\mathrm{V}$ shape actuator in the literature using numerical [8-11] and analytical [12-26] approaches. The advantage of the analytical modeling is in the better understanding of the actuator behavior and the influence of the different parameters on its performance. This leads to an intuitive 


\section{Task Feasibility of V Shape Electrothermal Actuators}

understanding on how the actuators dimensions can be varied to tune its properties according to the application.

Explicit analytical expressions are provided in this study governing the output forcedeflection behavior of the $\mathrm{V}$ shape actuator. These expressions are derived based on the snap-through modeling of pre-shaped beams [27], and show very good agreement with finite element simulations. The advantage of these expressions compared to the cited models is in the consideration of all internal and external parameters and phenomena that affect the actuator behavior in practice. This includes the response to electrical input, interaction with external lateral load, and buckling, which leads to a crucial variation in the stiffness of the actuator.

The geometry of the $\mathrm{V}$ shape actuator can be chosen based on the intuition of the designer and by trial and error. However, this does not lead to efficient and optimized performance. Few studies $[28,29]$ presented formal optimization procedure for improving certain characteristics of the $\mathrm{V}$ shape actuator. The design optimization of a $\mathrm{V}$ shape electrothermal actuator is investigated in this paper from task feasibility perspective and based on the governing expressions from the modeling.

Firstly, the influence of the material properties and geometrical dimensions on the actuator behavior is analyzed. Afterwards, several design constraints related to the limitations on the voltage, temperature, dimensions, and fabrication resolution are elaborated. These constraints haye to be considered in the design in order to ensure good fabrication and functioning of the actuator, and to avoid its failure. The task feasibility of the actuator is finally investigated.

The $\mathrm{V}$ shape actuator is generally fabricated in a micro- or nano-system to deal with one or limited number of loads. The versatility of changing the application once fabricated is not possible as for actuators at the macro-scale. Thus, a design-to-task approach is generally considered for the design of such actuators. From this perspective, relating the design to the specific task that the actuator is intended to deal with is a main concern.

In the task feasibility analysis in this paper, a performance index, named $Z$-value, is proposed for measuring the capacity of the actuator to provide the required forces and deflections. As it is developed based on the modeling expressions, the $Z$-value is also considering the relevant phenomena affecting the actuator behavior.

The rest of the paper is organized as follows. The force-deflection curve and the influence of the different parameters on the actuator performance are analyzed in Section 1. Several design constraints, which are necessary for a feasible design, are elaborated in Section 2. The task feasibility and optimization are finally investigated in Section 3. 
Task Feasibility of $V$ Shape Electrothermal Actuators

\section{Force-deflection behavior}

\subsection{Force-deflection curve}

Figure 1 shows top view schematics of a V shape electrothermal actuator in the initial configuration (Figure 1.a), electrically heated with free load (Figure 1.b), electrically heated with lateral load (Figure 1.c), and electrically heated with lateral load and zero displacement at the middle position (Figure 1.d). The actuator consists usually of several $\mathrm{V}$ shape beams connected in parallel to a middle shuttle. The shuttle is not shown in Figure 1 for clarity.

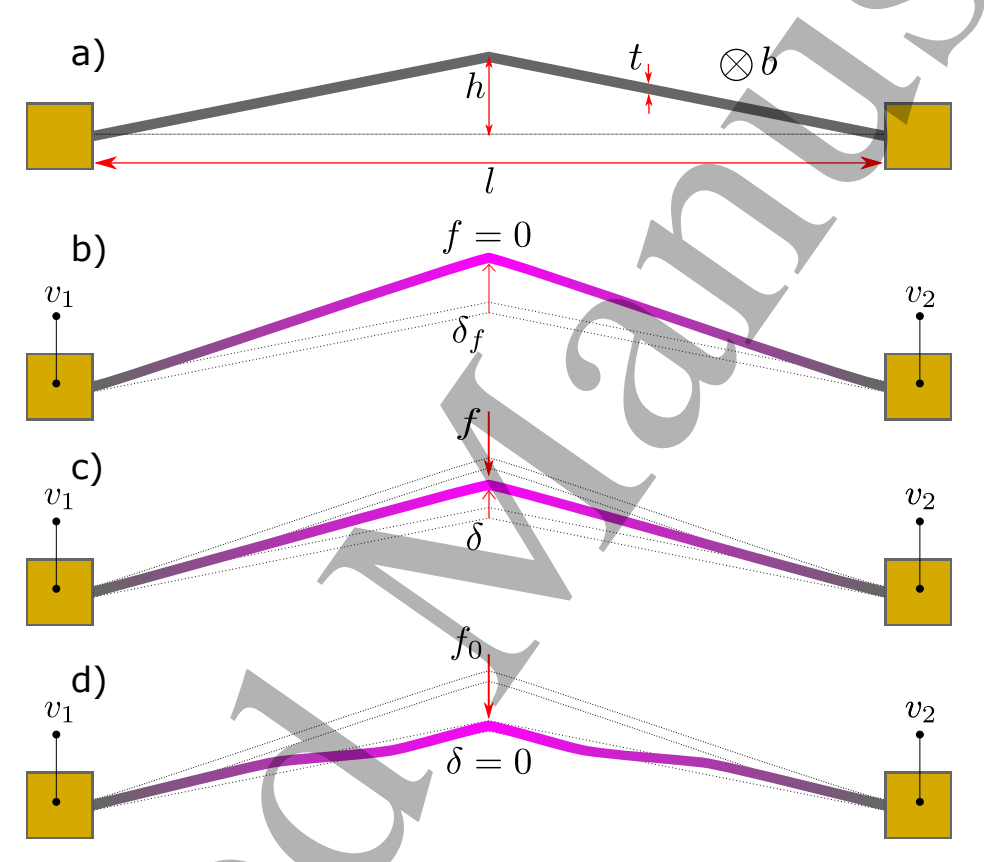

Figure 1. Schematics of a beam electrothermal actuator (V shape) in different configurations: initial (a), activated with free load (b), with lateral load (c), and at the zero deflection position (d).

The $\mathrm{V}$ shape actuator has a span $l$, in-plane thickness $t$, initial middle height $h$, and out-of-plane depth $b$. The voltage difference between boundaries $\left(\Delta v=v_{1}-v_{2}\right)$ is considered as the electrical input. The lateral deflection and load at the middle are denoted $\delta$ and $f$, respectively. $f$ can be seen as the external load or the pushing force generated by the actuator, as both are equal in magnitude. $\delta_{f}$ is the free load deflection due to heating $\left(\delta=\delta_{f}\right.$ for $\left.f=0\right)$, and $f_{0}$ is the zero deflection load $\left(f=f_{0}\right.$ for $\left.\delta=0\right)$. The actuator is usually used for pushing loads in the distance between $\delta=0$ and $\delta_{f}$. Buckling may occur in this range of deflection depending on the electrical input. The $f-\delta$ relationship without buckling in this range of interest is expressed as follows:

$$
\delta=h\left(\frac{2 \Lambda_{e}^{2}}{\Lambda_{a}}\left(\sqrt{1-\frac{\Lambda_{a}}{4 \Lambda_{e}^{2}} \frac{N^{2}-V^{2}}{12 Q^{2}}}-1\right)\right)
$$


Task Feasibility of V Shape Electrothermal Actuators

$$
f=\frac{E b t^{3}}{12 l^{3}}\left(4 h N^{2}+\frac{\delta}{\Lambda_{e}}\right)
$$

where $E$ is the Young's modulus, $N$ is a parameter related to the axial force, and $V$ is the normalized electrical input. $\Lambda_{a}$ and $\Lambda_{e}$ are functions of $N$ as follows:

$$
\begin{aligned}
& \Lambda_{a}(N)=\frac{3}{16 N^{4}}\left(1-\frac{\tan \frac{N}{4}}{\frac{N}{4}}+\frac{\tan ^{2} \frac{N}{4}}{3}\right) \\
& \Lambda_{e}(N)=\frac{1}{N^{3}}\left(\frac{N}{4}-\tan \frac{N}{4}\right)
\end{aligned}
$$

$V$ is related to the beam length expansion due to heating $\Delta s_{h}$, and is expressed as follows:

$$
V^{2}=12 \frac{l}{t^{2}} \Delta s_{h}
$$

Considering a straight beam electrothermal model with conduction (Appendix Appendix A), $V$ is approximated as follows:

$$
V^{2}=\frac{\Delta v^{2} \alpha l^{2}}{\rho_{0} K_{p} t^{2}}
$$

Each value of $N$ in (1) corresponds to one $(\delta, f)$ point. In the range of interest $\left(\delta \in\left[0, \delta_{f}\right]\right)$, the $f-\delta$ expression in (1) applies for $N \in\left[N_{f}, N_{0}\right]$, whereas $N$ increases monotonically from a minimum $N_{f}$ at free load position $\left(\delta=\delta_{f}\right)$ to a maximum $N_{0}$ at zero deflection position $(\delta=0) . N_{f}$ is in the range $[0,2 \pi]$, while $N_{0}=\min [V, 4 \pi]$.

Buckling occurs in the range of interest $\left(\delta \in\left[0, \delta_{f}\right]\right)$ for $V \geq 4 \pi$ (see Figure 1.d). The $f-\delta$ relationship with buckling is expressed as follows:

$$
f=\frac{16 \pi^{2}}{3} \frac{E b t^{3}}{l^{3}}(h+\delta)
$$

The governing $f-\delta$ expressions in (1) and (7) are derived from the modeling of pre-shaped beams [27] after considering axial expansion due to heating, as clarified in Appendix Appendix A. Analyzing these expressions, we can differentiate three $f-\delta$ curve forms depending on the value of $V$, as shown in Figure 2.

For $V$ lower than a certain limit $V_{m}$, the pushing force of the actuator decreases with the generated deflection. For higher electrical input $\left(V \geq V_{m}\right)$, the pushing force increases to a maximum and then starts to decrease until reaching the free load position. $V_{m}$ is the root of the following equation:

$$
\frac{V_{m}^{3}}{\frac{V_{m}}{4}-\tan \frac{V_{m}}{4}}=192 Q^{2} \quad 0 \leq V_{m} \leq 4 \pi
$$

where $Q$ is the height-to-thickness ratio $(Q=h / t)$.

For higher electrical input $(V \geq 4 \pi)$, buckling occurs in the range of deflection where the pushing force increases with deflection. From (8), we can deduce that the pushing force only decreases with the deflection without buckling for $Q>\pi / \sqrt{3}$. Thus, only two forms for the $f-\delta$ curves exist after this value of $Q$. 


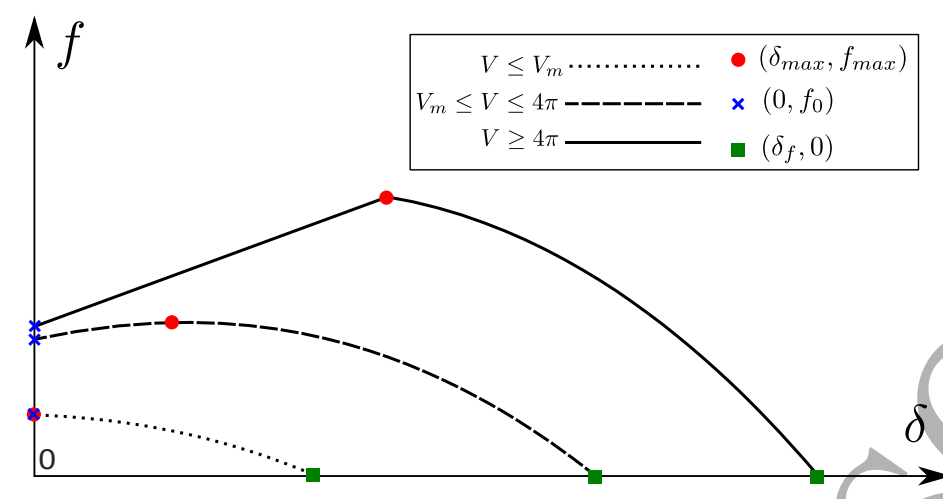

Figure 2. Force-displacement curves for the $\mathrm{V}$ shape actuator for different normalized electrical input $V$. For $V \leq V_{m}$, the output force decreases with further deflection. For $V_{m} \leq V \leq 4 \pi$, the output force increases slightly and then decreases with the deflection. For $V \geq 4 \pi$, the output force increases linearly in the buckling zone and then decreases with the deflection.

\subsection{Parameters variation influence}

Three points with 4 coordinates $\left(f_{0}, f_{\max }, \delta_{\max }\right.$, and $\left.\delta_{f}\right)$ are mainly constituting the $f-\delta$ curve. This curve shows the force-deflection output that can be provided by the actuator. The influence of the material properties and geometrical dimension on the actuator output is analyzed in this part.

Note that, as the $\mathrm{V}$ shape actuator is micro- or nano-fabricated, the springback, which is a common effect related to the fabrication of $\mathrm{V}$ shape structures at the macroscale $[30,31]$, is not considered as a parameter affecting the design.

1.2.1. Zero deflection force The pushing force at zero deflection $f_{0}$ can be deduced from (2) and (7):

$$
f_{0}=\frac{E b t^{3} h}{12 l^{3}} \cdot \begin{cases}4 V^{2} & V=[0,4 \pi] \\ 64 \pi^{2} & V>4 \pi\end{cases}
$$

The variation of $f_{0}$ with $V$ is shown in Figure 3.

1.2.2. Maximum force The maximum force point coordinates $\left(\delta_{\max }, f_{\max }\right)$ in the range $\left(\delta \in\left[0, \delta_{f}\right]\right)$ are expressed as follows:

$$
\begin{gathered}
\delta_{\max }= \begin{cases}0 & V \leq V_{m} \\
\delta_{m} & V_{m} \leq V \leq 4 \pi \\
h\left(-\frac{2}{3}+\frac{2}{3} \sqrt{\left.1-\frac{16 \pi^{2}-V^{2}}{16 Q^{2}}\right)}\right. & V \geq 4 \pi\end{cases} \\
f_{\text {max }}=\frac{E b t^{3} h}{12 l^{3}} \begin{cases}4 V^{2} & V \leq V_{m} \\
f_{m} & V_{m} \leq V \leq 4 \pi \\
64 \pi^{2}\left(\frac{1}{3}+\frac{2}{3} \sqrt{\left.1-\frac{16 \pi^{2}-V^{2}}{16 Q^{2}}\right)}\right. & V \geq V_{m}\end{cases}
\end{gathered}
$$




\section{Task Feasibility of $V$ Shape Electrothermal Actuators}

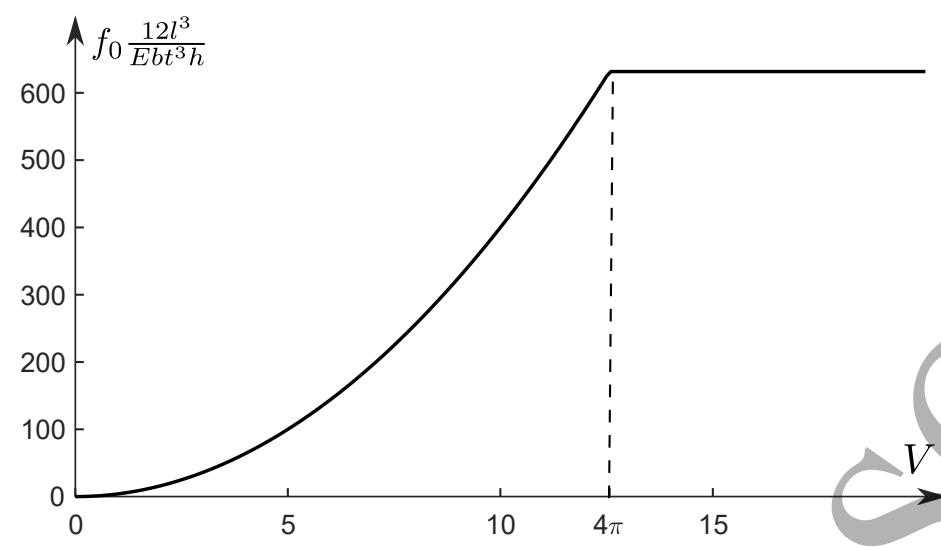

Figure 3. Variation of the zero deflection force $f_{0}$ with respect to $V . f_{0}$ increases monotonically with $V$ and becomes saturated for $V \geq 4 \pi$.

where $\left(\delta_{m}, f_{m}\right)$ are determined by substituting the value of $N(N \in[0,4 \pi])$ resulting in $\left(\frac{\partial f}{\partial \delta}=0\right)$ into $(1)$.

Figure 4 shows the variation of the maximum force $f_{\max }$ with respect to $V$ for different values of $Q$.

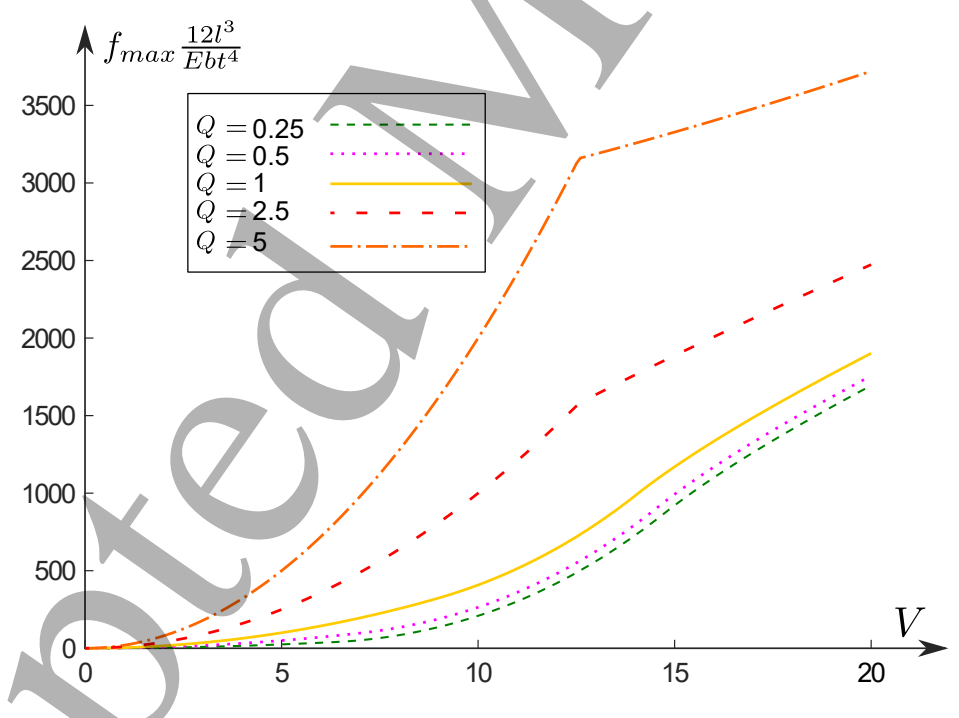

Figure 4. Variation of the maximum force $f_{\max }$ with respect to $V$ for different values of $Q$. For constant value of $t, f_{\max }$ increases with higher values of $Q$ and $h$, respectively.

Note that the vertical axes in Figures 3 and 4 are dependent mainly on the force quantity, but also dependent on dimension and material properties. For specific material and dimensions, these axes can be changed to show only the variation of the force quantity. We keep the parameters in the horizontal and vertical axes in Figure 4 independent from $h$. Thereby, the variation with $Q$ is proportional to the variation with $h$, while fixing the other dimensions. Hence, Figure 4 shows that $f_{\text {max }}$ increases with $h$, while keeping the other dimensions constant. 
For a fixed value of $t$ and low electrical input (low $V$ ), there is a certain value of $Q$ that maximizes $\delta_{f}$, as shown in the inset of Figure 5. For higher values of $V$, increasing $h$ while fixing the other dimensions results in reducing the free displacement of the actuator. The value of $Q$ that maximizes $\left(\delta_{f} / t\right)$ is calculated numerically for each value of $V$ and shown in Figure 6.

1.2.4. Summary of the parameters influence The force and deflection of the actuator increase generally with higher electrical input $V$ and thus with higher length-to-thickness ratio, since $V$ is proportional to $l / t(6)$. The actuator is stiffer with the Young's modulus $E$ and the output force is proportional to the width $b$. Both force and deflection outputs increase when increasing $t$ and $h$, while keeping $Q$ constant. However, they vary differently with respect to $Q$ and $l$. The force increases for higher $Q$, while it

Figure 5. Variation of the free deflection $\delta_{f}$ with respect to $V$ for different values of $Q$. The free deflection is higher with lower values of $Q$, except for low values of $V$, where the free deflection is higher for certain values of $Q$. The curves refer to different $Q$ values according to the legend in Figure 4.

Figure 5 shows variation of $\delta_{f}$ with respect to $V$ for different values of $Q$. Similarly to Figure 4, we keep the parameters in the horizontal and vertical axes in Figure 5 independent from $h$.

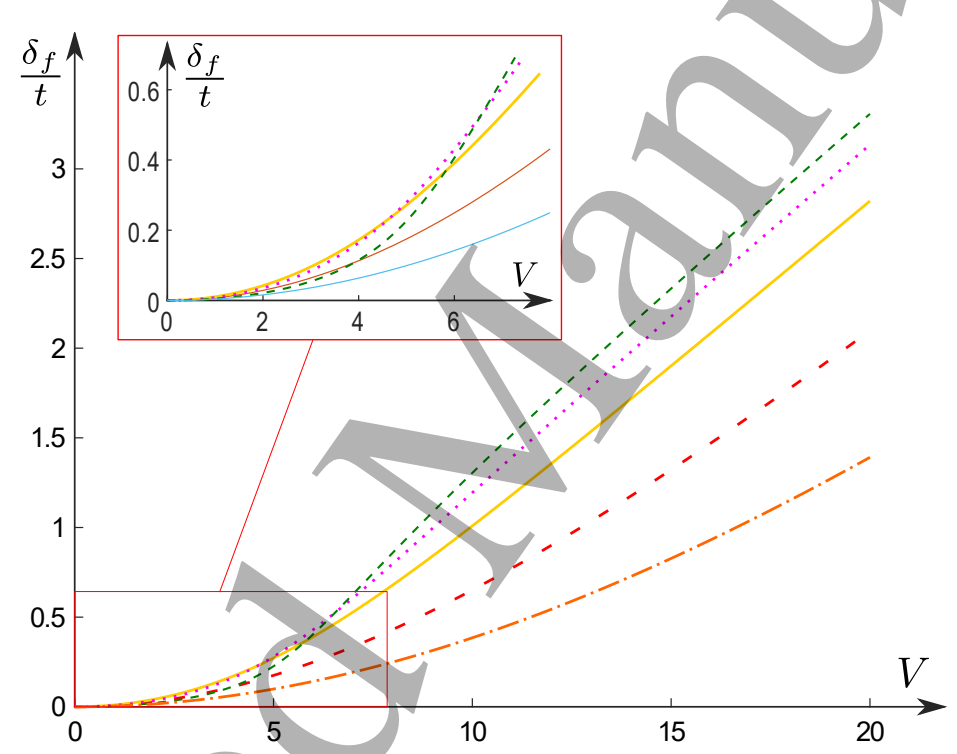




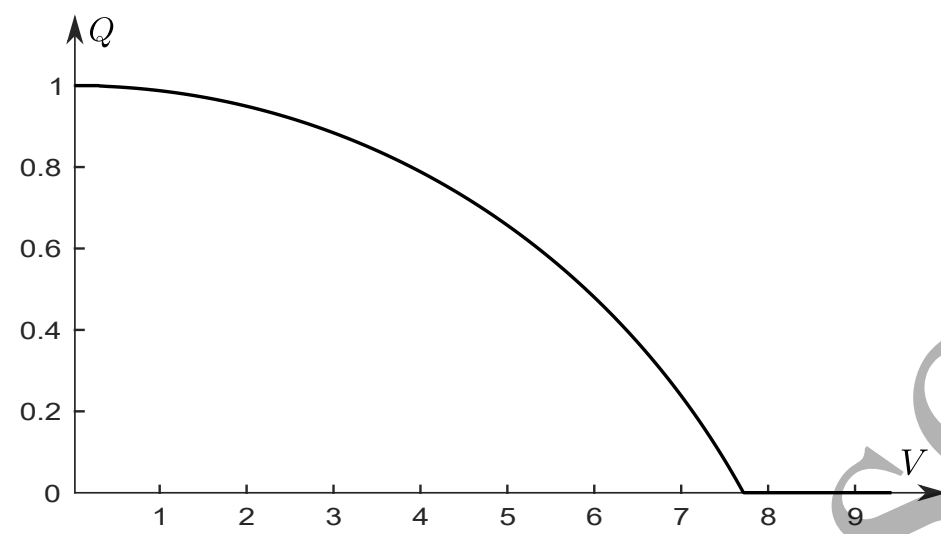

Figure 6. Variation of $Q$ value maximizing $\left(\delta_{f} / t\right)$ with respect to $V$. This value of $Q$ decreases with $V$, and becomes equal to zero at high values of $V$.

decreases for larger length. In contrast, the deflection decreases for higher $Q$, while it increases for larger length. For low value of $V$, the displacement is maximized at certain level of $Q$. Table 1 summarizes the influence for the variation of the Young's modulus and the dimensions on $f_{0}, f_{\text {top }}, \delta_{\text {top }}$, and $\delta_{f}$ on the $f-\delta$ points in the range of interest.

Table 1. Variation of the characteristic curve points with the Young's modulus and different dimensions.

\begin{tabular}{ccccccc}
\hline & \multicolumn{2}{c}{$f_{0}$} & $f_{\max }$ & $\delta_{\max }$ & $\delta_{f}$ \\
\cline { 2 - 6 } & $V<4 \pi$ & $V \geq 4 \pi$ & $V \geq 4 \pi$ & $V \geq 4 \pi$ & $V \gg$ \\
\hline \hline$E$ & $\nearrow^{1}$ & $\nearrow^{1}$ & $\nearrow$ & - & - \\
$b$ & $\nearrow^{1}$ & $\nearrow^{1}$ & $\nearrow$ & - & - \\
$t, h$ & $\nearrow^{2}$ & $\nearrow$ & $\nearrow$ & $\nearrow$ & $\nearrow$ \\
$Q$ & $\nearrow$ & $\nearrow$ & $\nearrow$ & $\searrow$ & $\searrow$ \\
$l$ & $\searrow$ & $\searrow^{3}$ & $\searrow^{2}$ & $\nearrow$ & $\nearrow$ \\
\hline
\end{tabular}

The arrow $\nearrow$ in Table 1 means that the corresponding force or deflection level increases with the increase of the corresponding property or dimension. The arrow $\searrow$ means the inverse. The index next to arrows (such as $\nearrow^{3}$ or $\searrow^{2}$ ) means that the corresponding force or displacement in the column varies with the index order of the corresponding property or dimension in the row. The row for " $t, h$ " shows the variation with respect to $t$ and $h$ while considering a constant ratio between them.

For very high values of $V(V \gg)$, the expressions for $f_{\max }, \delta_{\max }$, and $\delta_{f}$ can be further simplified as follows:

$$
f_{\text {max }} \approx \frac{8 \pi^{2}}{9} \frac{E b t^{4} V}{l^{3}} \quad \delta_{\text {max }} \approx \frac{t V}{6} \quad \delta_{f} \approx \frac{t V}{\pi \sqrt{3}}
$$




\section{Task Feasibility of $V$ Shape Electrothermal Actuators}

\section{Design constraints}

General restrictions on the actuator geometry and a maximum electrical input should be considered to avoid failure of the actuator during its functioning.

The actuator geometry is characterized by four parameters, including $b, t, h$, and $l$. $b, t$, and $l$ are strictly positive since these are dimensions for a filled-material structure. A zero value of $h$ means that the $V$ shape is actually a straight beam, where the direction of motion is not controllable after heating. Thus, we also limits $h$ to strictly positive values.

$$
b>0, \quad t>0, \quad h>0, \quad l>0
$$

The span $l$ is generally much higher than the other dimensions. This keeps valid the small deformation hypothesis considered in the structural modeling. Further, for large length-to-thickness ratio, the electrothermal input $V$ is higher and the actuator deflection/pushing force is amplified.

$$
l \gg b, t, h
$$

The fabrication resolution, at the macro-, micro-, or nano-scale, constraints a minimum feature size that can be fabricated properly. This makes a minimum limitation on the thickness $t$, being the smallest in-plane dimension for a material-filled-structure.

$$
t \geq t_{\min }
$$

The width $b$ corresponds generally to the thickness of the device layer in the chip or the wafer. The out-of-plane deformation is limited for sufficiently high value of $b$. The force provided by the actuator is proportional to $b$. However, if the actuator is dealing with other components in the same device layer, the value of $b$ is not critical for actuation since the forces from these components for in-plane motion (i.e. restoring force, electrostatic force, inertia force) are also proportional to $b$. In lithography-based fabrication (common in micro- and nano-fabrication), the minimum opening to be etched is usually equal to the minimum feature size, and both are limited by the maximum allowable aspect ratio (i.e. $b / t)$.

$$
\frac{b}{t} \leq \text { Max aspect ratio }
$$

In a design context, the maximum performance for the actuator is identified to ensure its capacity to provide the required force and deflection. According to the previous section, the maximum performance is reached considering the highest possible value of $V$.

One limitation in the functioning of the actuator is the maximum allowable temperature rise $\Delta T_{\max , a}$. This limit is related to the material capacity to support high temperature. The relation between the applied voltages and the maximum temperature rise $\Delta T_{\text {max }}$ induced in the actuator is approximated as follows:

$$
\Delta v=\sqrt{8 \rho_{0} K_{p} \Delta T_{\max }}
$$




\section{Task Feasibility of V Shape Electrothermal Actuators}

where (19) is calculated based on the electrothermal model of a straight beam [32].

Thereby, the maximum of $\Delta v$ and $V$ are defined based on the admissible temperature rise $\Delta T_{\max , a}$ :

$$
\begin{aligned}
& \Delta v_{\max }=\sqrt{8 \rho_{0} K_{p} \Delta T_{\max , a}} \\
& V_{\max }=\frac{l}{t} \sqrt{8 \alpha \Delta T_{\max , a}}
\end{aligned}
$$

Note that, as $\delta$ and $f$ are proportional to $V$ and $E V$ (14), respectively, a material with higher value of $\alpha \Delta T_{\max , a}$ is able to provide higher deflection, while a material having higher value of $E \sqrt{\alpha \Delta T_{\max , a}}$ is able to provide higher forces.

In addition to temperature, the stress inside the actuator should/not exceed a certain limit. The stress calculation is not in the scope of this paper. This can be checked with finite element simulations. However, if an actuator design shows high stress value during deflection in its range of interest, this can be relaxed by increasing the dimensional ratio $l / t[33]$.

The temperature and stress limits for the material have to be considered to avoid the fracture and failure of the actuator. A safety factor in the definition of these limits enhances the design robustness. For silicon, the fracture occurs at 2 GPa stress or higher at room temperature $[34,35]$. This varies depending on many parameters, including fabrication conditions, crystal orientation, geometry patterns, temperature, etc. [36]. A failure stress around $0.8 \mathrm{GPa}$ is measured on polysilicon structures at $800{ }^{\circ} \mathrm{C}$ in [37]. Silicon electrothermal actuators keep a proper functioning with temperature and stress limits up to $650^{\circ} \mathrm{C}$ and $0.5 \mathrm{GPa}$, respectively.

\section{Task feasibility}

The $\mathrm{V}$ shape actuator is generally designed to deal with a specific task, where a set of force/deflection outputs need to be provided by the actuator. However, there is a lack of a formal measure evaluating the capacity of the actuator to provide the required force/deflection outputs. A task feasibility measure for the $\mathrm{V}$ shape actuator is derived in this section. A case study is presented showing the capacity of the task feasibility measure to find a feasible configuration and to optimize other characteristics while ensuring task feasibility.

\subsection{Task feasibility measure}

The $f-\delta$ curves (Figure 2) visualize the performance capacity of the actuator in terms of force and deflection for a certain electrical input. The $f-\delta$ curve is called as the characteristic curve when considering the maximum electrical input (20). The characteristic curve shows the full performance capacity of the actuator. This means that the actuator is able to move any load $f_{r}$ a certain distance $\delta_{r}$, where the point $\left(\delta_{r}, f_{r}\right)$ lies inside the characteristic curve. Thereby, a design objective/constraint is to 
1

2

3

4

5

6

7

8

9

\section{Task Feasibility of V Shape Electrothermal Actuators}

expand the characteristic curve to include the required force-deflection points $\left(\delta_{r} \geq 0\right.$, $\left.f_{r} \geq 0\right)$.

Depending on the application, the required force and deflection can be defined at one or several discrete points or in a continuous force-deflection closed area. Figure 7 shows different required points $\left(r_{1}, r_{2}, r_{3}, r_{4}\right)$ with respect to the characteristic curve.

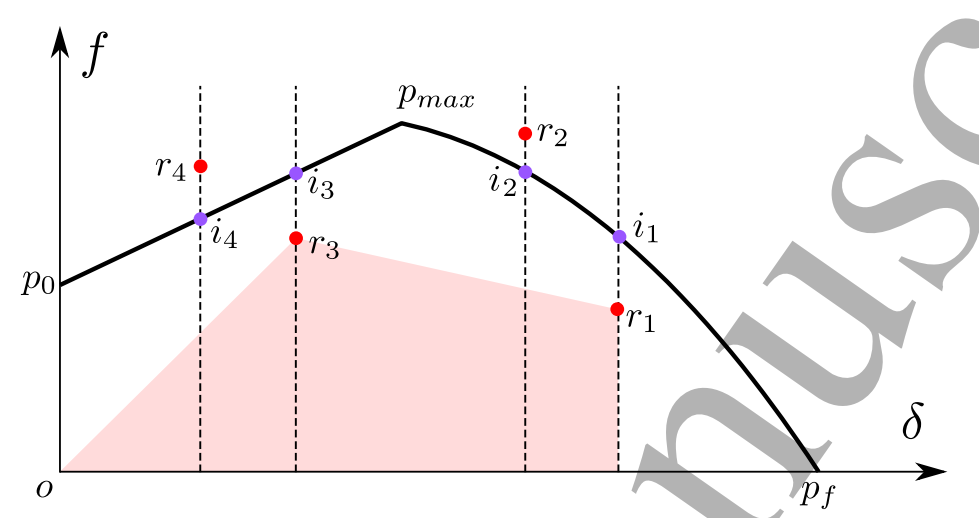

Figure 7. Required force-deflection points with respect to the characteristic curve. $o(0,0), p_{0}\left(0, f_{0}\right), p_{\max }\left(\delta_{\max }, f_{\max }\right)$, and $p_{f}\left(\delta_{f}, 0\right)$ are the origin, zero displacement point, maximum force point, and free-deflection point, respectively.

The points $r_{1}$ and $r_{2}$ in Figure 7 are in the no-buckling zone, while $r_{3}$ and $r_{4}$ are in the buckling zone. Let $i\left(\delta_{r}, f\left(\delta_{r}\right)\right)$ is the projection of a required point $r\left(\delta_{r}, f_{r}\right)$ on the characteristic curve with the same $\delta$ position. $i_{1}, i_{2}, i_{3}$, and $i_{4}$ are the projections of $r_{1}, r_{2}, r_{3}$, and $r_{4}$, respectively. Thereby, an actuator with characteristic curve as in Figure 7 is not able to provide required points $r_{2}$ and $r_{4}$, since $i_{2}$ and $i_{4}$ are below them, respectively. Hence, the feasibility of a point $\left(\delta_{r}, f_{r}\right)$ by the actuator can be directly checked as follows:

$$
\left\{\begin{array}{l}
\delta_{r} \leq \delta_{f} \\
f_{r} \leq f\left(\delta_{r}\right)
\end{array}\right.
$$

where $\delta_{f}$ is calculated from (12), while $f\left(\delta_{r}\right)$ is calculated from (1) when there is no buckling and from (7) in case of buckling. $\delta_{f}$ and $f\left(\delta_{r}\right)$ are calculated considering $V=V_{\max }$.

As explained in Section 1 , the buckling occurs for $V_{\max } \geq 4 \pi \& \delta_{r} \leq \delta_{\max }$, where $\delta_{\max }$ is calculated from (10) considering $V=V_{\max }$. Subsequently, the following $z$-value needs to be negative to satisfy task feasibility for one required force-deflection point $\left(\delta_{r}, f_{r}\right)$ :

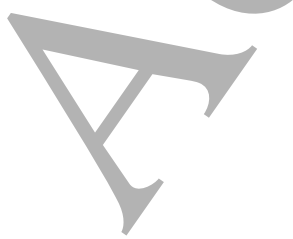

if $V_{\max } \geq 4 \pi \& \delta_{r} \leq \delta_{\max }$

$z=f_{r}-\frac{16 \pi^{2}}{3} \frac{E b t^{3}}{l^{3}}\left(h+\delta_{r}\right)$

else if $\delta_{r} \leq \delta_{f}$

$$
z=f_{r}-\frac{E b t^{3}}{12 l^{3}}\left(4 h N^{2}+\frac{\delta_{r}}{\Lambda_{e}(N)}\right)
$$

else

$$
z=\delta_{r}-\delta_{f}
$$




\section{Task Feasibility of V Shape Electrothermal Actuators}

where $N$ is calculated from (1) with $\delta=\delta_{r}$ and $V=V_{\max }$.

Minimizing the $z$-value by changing the actuator dimensions leads to increasing $\delta_{f}$ in case $\delta_{r}>\delta_{f}$, or otherwise to increase the force provided by the actuator at the required position $\delta_{r}$. In both cases, the characteristic curve tends to expand to contain the required point. The $z$-value becomes negative when the required point $\left(\delta_{r}, f_{r}\right)$ is included in the characteristic curve.

In case the actuator is required to deal with several loads at different positions (i.e. several required points), the $z$-value (23) needs to be satisfied for each required point. We define $Z$ as the maximum of $z$ considering the different required points. Accordingly, $Z$ needs to be negative to satisfy the required force-deflection points:

$$
Z=\max _{i}\left(z_{i}\right)
$$

where $z_{i}$ is the $z$ value (23) for the $i^{\text {th }}$ required point.

Note that, if two points $r_{i}$ and $r_{j}$ are required, $r_{j}$ lies under the line segment $\left[o r_{i}\right]$, then $r_{j}$ is necessarily feasible if $r_{i}$ is feasible (i.e. the actuator is able to provide $r_{j}$ if it is able to provide $r_{i}$ ). Further, as the area below the characteristic curve for positive $\delta$ and $f$ (i.e. $o p_{0} p_{\max } p_{f}$ ) is convex, if several points are included in this area, then the convex hull of these points is also included in the same area. The origin point $o$ can be considered as a feasible point independently from the actuator performance. Considering these hypotheses, if several points are feasible (including the origin), then their convex hull in addition to the area underneath are also feasible. This can be seen in Figure 7 , where the red area is feasible as far as $o, r_{1}$, and $r_{3}$ are feasible.

Based on the aforementioned, the task feasibility measure is the $Z$-value (24), which is a maximum of the $z$-value calculated from (23) for each required $f-\delta$ point. This measure can be used to find feasible and optimized configurations for the $\mathrm{V}$ shape actuator, as shown in the next parts.

3.1.1. Optimization Optimization algorithms search for an optimal solution in the space of feasible solutions. Starting an optimization from outside this space does not guarantee finding a feasible solution. Thus, optimization requires the knowledge of an initial feasible configuration, i.e. respecting the different constraints in terms of dimensions and task feasibility.

Based on the feasibility analysis in the previous part, a feasible configuration can be found by minimizing the $Z$ value while varying the geometry within the dimension limits and considering the material properties.

$$
\min _{b, t, h, l} \text { such that }\left\{\begin{array}{l}
Z \\
b, t, h, l \in \text { dimension limits } \\
\text { material properties }
\end{array}\right.
$$

The minimization in (25) can be carried using optimization software. A feasible configuration for the actuator can be found, if the minimization in (25) can lead to a configuration with negative value of $Z$. Otherwise, if (25) does not lead to a negative 


\section{Task Feasibility of $V$ Shape Electrothermal Actuators}

value of $Z$, no feasible configuration can be found within the range of dimensions allowed for the actuator. Recalling that $f_{r}$ is the force required from a single beam of the actuator. If several beams are connected together in parallel, $f_{r}$ is the total required force divided by the number of beams. Increasing the number of actuator beams in parallel is an option if higher forces are required.

Once a feasible configuration for the actuator is obtained, optimizing other aspects relevant to the actuator can be carried, while respecting the dimension limits and the task feasibility $(Z \leq 0)$. Depending on the application, several objectives can be targeted in the design optimization. The design optimization can target size miniaturization, maximizing output force and deflection, minimizing entropy, faster time response, etc.. The objective function is defined accordingly and the optimization is formulated as follows:

$$
\begin{array}{ll}
\underset{b, t, h, l}{\operatorname{optimize}} & \text { objective function } \\
\text { such that } & \left\{\begin{array}{l}
Z \leq 0 \\
b, t, h, l \in \text { dimension limits } \\
\text { material properties }
\end{array}\right.
\end{array}
$$

3.1.2. Case study A case study is presented showing the capacity of the design tools investigated in this study to optimize an actuator characteristic, while satisfying the task feasibility and different constraints. The design objective in the case study is to determine the minimum thickness $t$ for a $\mathrm{V}$ shape actuator while dealing with a high load $(4 \mathrm{mN})$ at small distance $(20 \mu \mathrm{m})$ and smaller load $(2 \mathrm{mN})$ at farther distance $(30 \mu \mathrm{m})$ (i.e. required force-displacement points are $r_{1}(20 \mu \mathrm{m}, 4 \mathrm{mN})$ and $\left.r_{2}(30 \mu \mathrm{m}, 2 \mathrm{mN})\right)$. The following parameters are considered: silicon material with $E=169 \mathrm{GPa}$, temperature dependent $\alpha$ [38], $\Delta T_{\max , a}=600^{\circ} \mathrm{C}, T_{\infty}=20^{\circ} \mathrm{C}$, fixed width and length $b=25 \mu \mathrm{m}$ and $l=2 \mathrm{~mm}$, and minimum feature size of $5 \mu \mathrm{m}$.

Considering these parameters, the design optimization can vary only $t$ and $h$. Starting from an initial guess of $t=10 \mu \mathrm{m}$ and $h=10 \mu \mathrm{m}$, we can deduce from the $\mathrm{Z}$ value (24) that the actuator is not able to provide the require points $r_{1}$ and $r_{2}$. Thus, a feasible configuration is firstly explored with the method defined in (25).

$$
\text { such that }\left\{\begin{array}{c}
Z \\
\min _{t, h}
\end{array} \quad \begin{array}{l}
r_{1}(20 \mu \mathrm{m}, 4 \mathrm{mN}), r_{2}(30 \mu \mathrm{m}, 2 \mathrm{mN}) \\
b=25 \mu \mathrm{m}, l=2 \mathrm{~mm}, t \geq 5 \mu \mathrm{m}, h>0 \mu \mathrm{m} \\
E=169 \mathrm{GPa}, \alpha \text { of silicon }[38] \\
\Delta T_{\max , a}=600^{\circ} \mathrm{C}, T_{\infty}=20^{\circ} \mathrm{C}
\end{array}\right.
$$

The value of $t$ and $h$ obtained after running the optimization in (27) are $t=$ $23.76 \mu \mathrm{m}$ and $h=11.74 \mu \mathrm{m}$. The $\mathrm{Z}$ value for this configuration is negative. This indicates that there are feasible configurations for the actuator, respecting the design constraints. 


\section{Task Feasibility of V Shape Electrothermal Actuators}

Afterwards, the thickness minimization is run starting from the configuration obtained in $(27)$.

$$
\min _{t, h} \text { such that }\left\{\begin{array}{l}
Z \leq 0 \\
r_{1}(20 \mu \mathrm{m}, 4 \mathrm{mN}), r_{2}(30 \mu \mathrm{m}, 2 \mathrm{mN}) \\
b=25 \mu \mathrm{m}, l=2 \mathrm{~mm}, t \geq 5 \mu \mathrm{m}, h>0 \mu \mathrm{m} \\
E=169 \mathrm{GPa}, \alpha \text { of silicon }[38] \\
\Delta T_{\max , a}=600^{\circ} \mathrm{C}, T_{\infty}=20^{\circ} \mathrm{C}
\end{array}\right.
$$

The value of $t$ and $h$ obtained from the optimization in $(28)$ are $t=14.60 \mu \mathrm{m}$ and $h=26.20 \mu \mathrm{m}$. The optimizations in (27) and (28) are carried out using MATLAB software based on sequential quadratic programming algorithm. Figure 8 shows the characteristic force-displacement curve for the initial configuration, minimum $Z$ configuration (27) and the optimal configuration with minimum thickness (28).

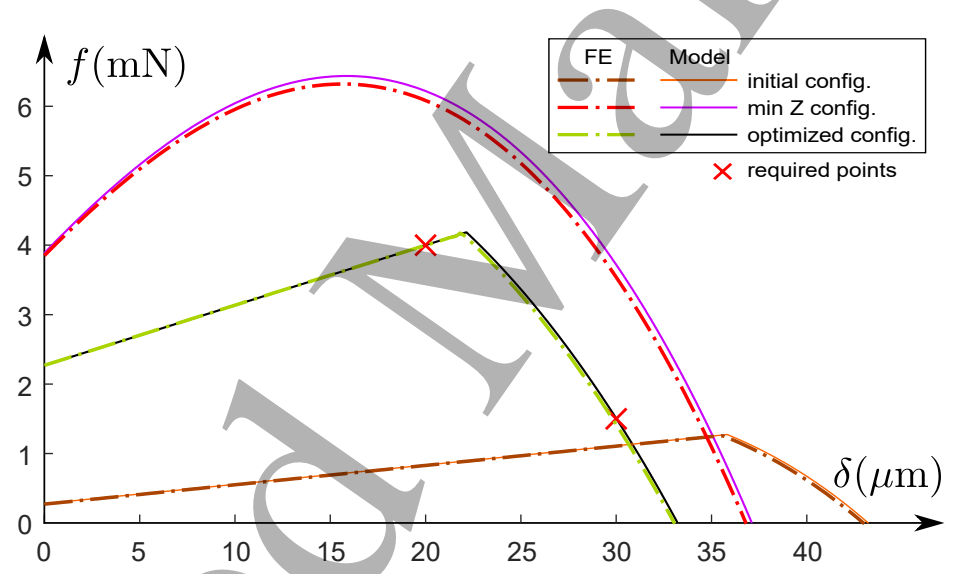

Figure 8. Characteristic force-displacement curve for the initial configuration, minimum $Z$ configuration and the optimal configuration. The characteristic curves are obtained from the analytical model and finite element (FE) simulations.

The characteristic curves in Figure 8 are obtained from the analytical expressions (i.e. (1) and (7)) and from finite element simulations using ANSYS software. The simulations on ANSYS are based on static analysis considering nonlinear large deformation and default solver type. The meshing is based on a tetrahedron planar element type (PLANE223) allowing a coupled structural-thermoelectric field analysis, with a fine mesh size defined with smart sizing function (level 2). The simulations are made in subsequent steps, starting by applying small deflection steps (up to $\delta>\delta_{f}$ ), then increasing the applied voltages to $\Delta v_{\max }$ with small voltage steps, and finally applying small deflection steps backwards to the initial position $\delta=0$. This allows simulating the buckling without divergence issues.

The comparison in Figure 8 shows a very good agreement between the analytical expressions and finite element simulations. Besides, Figure 8 shows that the required points are inside the characteristic curves for the minimum $Z$ and optimal configurations, 
while it is not the case for the initial configuration. This shows the usefulness and utility of the task feasibility measure provided in this study.

\section{Conclusion}

A formal task feasibility measure is derived for the design optimization of $\mathrm{V}$ shape electrothermal actuator. The governing analytical force-deflection expressions are firstly derived. The variation of the force and deflection with the geometry and material properties is then analyzed. General restrictions on the dimensions and electrical input are also elaborated to ensure good functioning without failure. The task feasibility measure is then proposed based on the force-deflection analysis for finding a feasible configuration and as a constraint for design optimization.

The modeling and design elements developed in this study provide a complete understanding of the actuator behavior, show the capacity/ and limitations of the actuator, and give insights on how the design can be changed to accomplish different tasks depending on the application.

\section{References}

[1] R Cragun L L H 1999 Linear thermomechanical microactuators uta.edu p 8

[2] Potekhina A and Wang C 2019869 ISSN 20760825

[3] Zhu Y, Moheimani S O R and Yuce M R 2012 IEEE Sensors Journal 12 2508-2509 ISSN 1530$437 \mathrm{X}$

[4] Pac M R and Popa D O 20113-DOF untethered microrobot powered by a single laser beam based on differential thermal dynamics Proceedings - IEEE International Conference on Robotics and Automation pp 121-127 ISBN 9781612843865 ISSN 10504729

[5] Zhang R, Sherehiy A, Yang Z, Wei D, Harnett C K and Popa D O 2019 ChevBot-An untethered microrobot powered by laser for microfactory applications Proceedings - IEEE International Conference on Robotics and Automation vol 2019-May (Institute of Electrical and Electronics Engineers Inc.) pp 231-236 ISBN 9781538660263 ISSN 10504729

[6] Steiner H, Hortschitz W, Stifter M, Keplinger F and Sauter T 2014 Thermal actuators featuring large displacements for passive temperature sensing Microsystem Technologies vol 20 (Springer Verlag) pp 551-557 ISSN 09467076

[7] Cohen Y and Ya'Akobovitz A 2019 IEEE Transactions on Instrumentation and Measurement 68 3766-3775 ISSN 15579662

[8] Lott C D, McLain T W, Harb J N and Howell L L 2002 Sensors and Actuators, A: Physical 101 239-250 ISSN 09244247

[9] Teichert K and Jensen B 2008 Sensors and Actuators, A: Physical 148 168-175 ISSN 09244247

[10] Nguyen D T, Hoang K T and Pham P H 2020 Heat Transfer Model and Critical Driving Frequency of Electrothermal V-Shaped Actuators Lecture Notes in Networks and Systems vol 104 (Springer) pp 394-405

[11] Vamegh Estahbanati S, Dhaouadi R and Bakri-Kassem M 2017 Mechatronics 46 193-204 ISSN 0957-4158

[12] Gianchandani Y B and Najafi K 1996 Journal of Microelectromechanical Systems 5 52-58 ISSN 10577157

[13] Sinclair M J 2000 Thermomechanical Phenomena in Electronic Systems -Proceedings of the Intersociety Conference 1 127-132 
Task Feasibility of $V$ Shape Electrothermal Actuators

[14] Enikov E T, Kedar S S and Lazarov K V 2005 Journal of Microelectromechanical Systems 14 788-798 ISSN 10577157

[15] Guan C and Zhu Y 2010 Journal of Micromechanics and Microengineering 20085014 ISSN 09601317

[16] Andò B, Baglio S, Savalli N and Trigona C 2011 IEEE Transactions on Instrumentation and. Measurement 60 1348-1357 ISSN 00189456

[17] Holst G L, Teichert G H and Jensen B D 2011 J Mech Design 133051002 ISSN 10500472

[18] 2012 Thermal Actuators Encyclopedia of Nanotechnology (Dordrecht: Springer Netherlands) pp 2680-2697

[19] Kwan A M H, Song S, Lu X, Lu L, Teh Y K, Teh Y F, Chong E W C, Gao Y, Hau W, Zeng F, Wong M, Huang C, Taniyama A, Makino Y, Nishino S, Tsuchiya T and Tabata O 2012 Journal of Microelectromechanical Systems 21 586-595 ISSN 10577157

[20] Li X, Zhao Y, Hu T, Xu W, Zhao Y, Bai Y and Ren W 2015 Microsystem Technologies 21 2367-2374 ISSN 09467076

[21] Zhang Z, Yu Y, Liu X and Zhang X 2015 A comparison model of V- and Z-shaped electrothermal microactuators 2015 IEEE International Conference on Mechatronics and Automation, ICMA 2015 (Institute of Electrical and Electronics Engineers Inc.) pp 1025-1030 ISBN 9781479970964

[22] Zhang Z, Yu Y, Liu X and Zhang X 2016 Dynamic electro-thermal modeling of V- and Zshaped electrothermal microactuator 2016 IEEE International Conference on Mechatronics and Automation, IEEE ICMA 2016 (Institute of Electrical and Electronics Engineers Inc.) pp 890895 ISBN 9781509023943

[23] Zhang Z, Yu Y, Liu X and Zhang X 2017 Microsystem Technologies 23 3775-3789 ISSN 09467076

[24] Aravind T, Ramesh R, Kumar S P and Ramya S 2018 Comparative Study of Different Materials on Performance of Chevron Shaped Bent-Beam Thermal Actuator Communications in Computer and Information Science vol 837 (Springer Verlag) pp 743-751 ISBN 9789811319358 ISSN 18650929

[25] Thangavel A, Rengaswamy R, Sukumar P K and Sekar R 2018 Microsystem Technologies 24 1767-1774 ISSN 09467076

[26] de S Gama G R, Coelho C A A, Gaspar J, Freitas P J P, Sommer R L and Mello A 2020 Journal of Microelectromechanical Systems 1-10 ISSN 1057-7157

[27] Hussein H and Younis M I 2020 Journal of Mechanisms and Robotics 12 ISSN 1942-4302 041001

[28] Długosz A, Jarosz P and Schlieter T 2019 Applied Sciences 94654 ISSN 2076-3417

[29] Osman S E and Zarog M 2019 Micro and Nanosystems 11 62-67 ISSN 18764029

[30] Aminzahed I, Mashhadi M M and Sereshk M R V 2017 Materials Science and Engineering: C 71 $685-689$

[31] Liu W, Zou X, Huang S and Lei Y 2019 The International Journal of Advanced Manufacturing Technology $100721-727$

[32] Lin L and Chiao M 1996 Sensors and Actuators, A: Physical 55 35-41 ISSN 09244247

[33] Hussein H, Bouhadda I, Mohand-Ousaid A, Bourbon G, Le Moal P, Haddab Y and Lutz P 2018 Sensors and Actuators A: Physical 271 373-382

[34] McLaughlin J C and Willoughby A F 1987 Journal of Crystal Growth 85 83-90 ISSN 00220248

[35] LaVan D, Jackson K, McKenzie B, Glass S, Friedmann T, Sullivan J and Buchheit T 2008 Direct Tension and Fracture Toughness Testing Using the Lateral Force Capabilities of a Nanomechanical Test System Mechanical Properties of Structural Films (ASTM International) pp $62-62-10$

[36] DelRio F W, Cook R F and Boyce B L 2015 Applied Physics Reviews 2021303 ISSN 1931-9401

[37] Boyce B L, Grazier J M, Buchheit T E and Shaw M J 2007 Journal of Microelectromechanical Systems 16 179-190 ISSN 10577157

[38] Okada Y and Tokumaru Y 1984 Journal of Applied Physics 56 314-320 ISSN 0021-8979 


\section{Task Feasibility of $V$ Shape Electrothermal Actuators}

\section{Appendix A. Derivation of governing expressions}

The expressions governing the variation of the actuator output force $f$ with the deflection $\delta$ is given in (1) for the case of no-buckling and in (7) for case of buckling. Both $f-\delta$ governing expressions in (1) and (7) apply in the range of actuation between zero deflection position $(\delta=0)$ and free load position due to heating $\left(\delta=\delta_{f}\right)$.

These expressions can be derived from the modeling study for the snap-through of pre-shaped beams (without being heated) developed in [27]. Due to electrothermal heating, an additional length expansion due to heating $\Delta s_{h}$ is added in the calculation of the axial force $P$. The latter can be calculated with the Hook's law as follows:

$$
P=E b t\left(\frac{\Delta s_{h}+\bar{s}-s}{\bar{s}}\right)
$$

where $s$ is the beam length and $\bar{s}$ is the initial beam length.

The length expansion $\Delta s_{h}$ is calculated as follows:

$$
\Delta s_{h}=\int_{0}^{l} \alpha(T(x, t)-\bar{T}(x)) d x
$$

where $\alpha$ is the thermal expansion coefficient of the material.

Considering a straight beam electrothermal model with conduction [32], $\Delta s_{h}$ can be approximated as follows:

$$
\Delta s_{h}=\frac{\Delta v^{2} \alpha l}{12 \rho_{0} K_{p}}
$$

where $K_{p}$ is the conduction coefficient, and $\rho_{0}$ is the electrical resistivity.

Considering (A.1), the $f-\delta$ expressions in (1) and (7) can be derived by following the same procedure as in [27]. 